\title{
Optical Underwater Communication: The Potential of Using Converted Green LEDs in Coastal Waters
}

\author{
Jan Sticklus ${ }^{\circledR}$, Member, IEEE, Peter Adam Hoeher $\left.{ }^{(}\right)$, Fellow, IEEE, and Rüdiger Röttgers
}

\begin{abstract}
Compared to the open ocean where blue lightemitting diodes (LEDs) perform well for data communications, in coastal and harbor environments optical transmission becomes worse and the color of lowest attenuation shifts to green. Another problem concerns the "green-yellow gap" of LEDs, as the quantum efficiency of current commercially available green LEDs is poor. Since energy consumption is an important factor, particularly for battery-powered systems, using blue LEDs is a tradeoff. Recently, phosphor-converted green LEDs, which are pumped by highly efficient blue LEDs, have been introduced to the market, and this type of LED promises better performance. This paper compares the use of blue, green, and converted green LEDs for applications in optical underwater communication systems in coastal waters. Theoretical aspects of the overall LED-water-detector channel are presented. A method for determining individual system coefficients is developed, and the impact on the communication system performance is explained. Practical approaches are introduced, complemented by measurements in the Baltic Sea.
\end{abstract}

Index Terms-Coastal waters, converted light-emitting diode (LED), green-yellow gap, optical underwater (u/w) communication.

\section{INTRODUCTION}

I $\mathrm{N}$ CONTRAST to commonly used acoustic underwater $(\mathrm{u} / \mathrm{w})$ communications, the optical $\mathrm{u} / \mathrm{w}$ channel promises much faster data transmission, but with the disadvantage of an orders of magnitude decreased range. Possible applications are autonomous $\mathrm{u} / \mathrm{w}$ vehicles operating in a swarm or single vehicles communicating with $\mathrm{u} / \mathrm{w}$ platforms, such as sensor landers. The basic components of $\mathrm{u} / \mathrm{w}$ optical links include a modulated light source at the transmitter side, the water column, and a photodetector at the receiver side. The modulated electrical signal needs to be converted into photons before traveling through the water column. Typical light sources that allow fast switching are lasers and light-emitting diodes (LEDs). A collimated laser beam provides high irradiance, offering a longer range compared to diffuse light sources, but requires sophisticated pointing and

Manuscript received December 21, 2016; revised September 25, 2017 and January 14, 2018; accepted March 9, 2018. Date of publication May 2, 2018; date of current version April 12, 2019. This work was supported by the Helmholtz Alliance "Robotic Exploration of Extreme Environments" (ROBEX). (Corresponding author: Jan Sticklus.

Associate Editor: J. Jaffe.

J. Sticklus is with the GEOMAR Helmholtz Centre for Ocean Research Kiel, Kiel 24148, Germany (e-mail: jsticklus@geomar.de).

P. A. Hoeher is with the Faculty of Engineering, University of Kiel, Kiel 24118, Germany (e-mail: ph@tf.uni-kiel.de).

R. Röttgers is with the Helmholtz Zentrum Geesthacht Centre for Materials and Coastal Research, Geesthacht 21502, Germany (e-mail: ruediger. roettgers@hzg.de).

Digital Object Identifier 10.1109/JOE.2018.2816838 acquisition when used with hovering or moving vehicles such as autonomous underwater vehicles (AUVs) or remotely operated vehicles. Therefore, for practical purposes, LEDs are a suitable compromise as their typically wide radiation pattern can be altered by reflectors or lenses. Our focus for setting up u/w optical systems is based on using commercially available components which are moderately priced and off the shelf. Widely used detectors at the receiver side are silicon photodiodes (PD), rather than expensive and fragile photomultiplier tubes, which would be the best choice for maximum ranges in the clear deep sea.

Developing $\mathrm{u} / \mathrm{w}$ systems requires testing before operation in the field. In most instances, deep sea gear testing is split into pressure tests for maximum depth and field trails in shallow waters for functionality checks, since the use of testing tanks have many limitations with respect to acoustics and optics. Baltic Sea $\mathrm{u} / \mathrm{w}$ tests allow us to reference the values from our setup to a commercially available oceanographic instrument. This referencing is beneficial, considering that this will also be the environment for investigating optically communicating AUVs in the future. LED-based mobile $\mathrm{u} / \mathrm{w}$ communication benefits from a diffuse (e.g., spherical) character of both transmitting and receiving elements. As the body of the vehicles often causes shadowing, two hemispherical optical transducer heads are common, analogous to lower and upper transducers in acoustics. For many optical u/w applications, pressure housings with a flat or hemispherical port made of glass or plastics are used. Generally, these housings are relative heavy, bulky, and expensive. The main disadvantage of the hemispherical port is the focus-sensitive layout, whereas the limited field of view (FOV) of up to $\pm 47^{\circ}$ [1] is the main drawback of the flat port design. Both designs suffer from numerous transitions through different media in the optical path, which may also cause uncertainties in analytic calculations. An alternative is to use pressure-neutral casting of optical components in a transparent resin. Many electronic components are able to withstand high pressure in deeper water. For field testing, we decided to cast LEDs and PDs in highly transparent polyurethane. Arranging several of these rigid elements allows the setup of a transducer head with a hemispherical characteristic, and also individual addressing of elements to save valuable energy. A possible close-to a hemispherical design is a truncated pyramid structure [2]. The number of elements used is a compromise between transmitted power, efficiency, space, and cost.

In this paper, we examine the usability of blue and green LEDs with the aim to support future developments in optical communication for $\mathrm{u} / \mathrm{w}$ vehicles in coastal waters. We present 
and discuss the main elements of the $\mathrm{u} / \mathrm{w}$ communication channel, beginning with the medium water in Section II, the light source in Section III, and the photodetector in Section IV. In addition, we also consider the effects of two typical modulation schemes in Section V. The experimental setup and procedures are introduced in Section VI, followed by the experimental results in Section VII. Finally, concluding remarks are provided in Section VIII.

\section{Optical Properties of SeAwATER}

For optical u/w communications, coastal waters, such as the Baltic Sea, are more challenging than oceanic waters. This is due to much higher complexity with respect to their inherent optical properties (including light absorption, scattering, and attenuation). The expected maximum range for optical $\mathrm{u} / \mathrm{w}$ communication in coastal waters is a few meters, thus it is one order of magnitude less than in the clear ocean [3]. There are two basic parameters describing the attenuation of light in water. For collimated light, it is the beam attenuation coefficient $c$, and for diffuse light it is the diffuse attenuation coefficient $K$, or $K_{d}$ for downwelling natural irradiance. For laser light, the use of $c$ is appropriate, but for less focused beams $K$ is more suitable [4]. While $c$ describes the inherent optical properties of the water and depends only on the medium, $K$ describes an apparent optical property, as $K$ depends on the medium and the radiance distribution, where the radiance distribution is determined mainly by the sun's position at the sky [5]. The parameter $K$ is upper limited by $c$, but is usually lower than $c$ as the typical radiance distribution is not that of a light beam. For clear oceanic water, $K$ is roughly a third part of $c$ for a given wavelength [3]. Depending on the application, a suitable attenuation coefficient value is found between $c$ and $K$ [3], [6]. The basic formulas for the decay of light intensity in water are

$$
E=E_{0} \cdot e^{-c \cdot r}
$$

and accordingly

$$
E=E_{0} \cdot e^{-K \cdot r}
$$

where $E_{0}$ is the initial irradiance (in watts per square meter). After traveling a distance $r$ (in meters) in a medium with an attenuation coefficient of $c$ or $K$ (in $1 / \mathrm{m}$ ), the residual irradiance is $E$ (in watts per square meter) [3]. Oceanic and coastal waters can be classified as the so-called Jerlov water types, starting with types I-III for the ocean and $1 \mathrm{C}-10 \mathrm{C}$ for coastal waters, varying from clear to more turbid [7]. Diffuse attenuation coefficients for Jerlov coastal water types $1 \mathrm{C}-9 \mathrm{C}$ and oceanic type III are shown in Fig. 1. The coefficients $K_{d}$ and $c$ are wavelength dependent, and the wavelength spectra of $K_{d}$ for different water types show individual minima at certain wavelengths. The minima shift from blue wavelengths for less turbid oceanic to green wavelengths for more turbid coastal waters. For harbor waters with high chlorophyll concentrations, red light was found to be least attenuated [8], [9]. For the Baltic Sea, $K_{d}$ values of types III to $9 \mathrm{C}$ are documented, depending on the region and season. Types 3C and 5C occur relatively often in the Baltic Sea [10]. As an inherent property, the attenuation coefficient $c$ is composed of

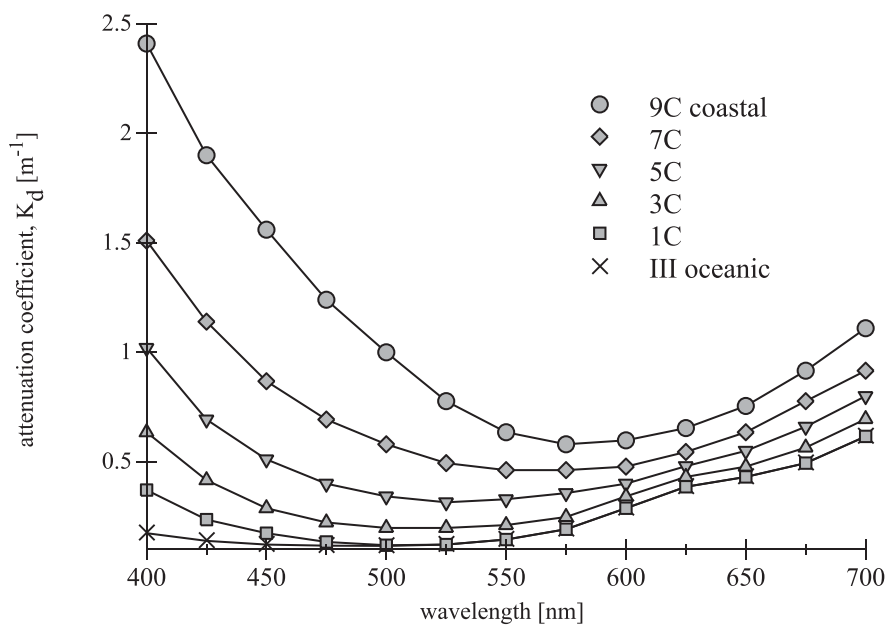

Fig. 1. Total diffuse light attenuation coefficients $K_{d}$ for the Jerlov water types (shown in the legend) as a function of light wavelength, replotted from database [7].

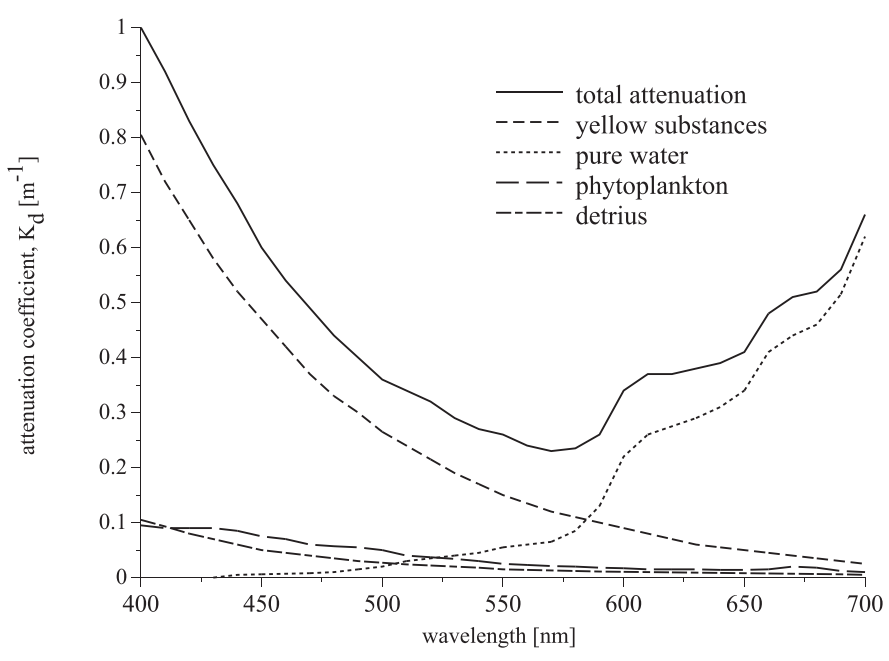

Fig. 2. Typical wavelength spectrum of the total diffuse attenuation coefficient $K_{d}$ for the Baltic Sea in summer (solid line). Additionally, the coefficients for different water constituents are given (see legend). Redrawn from [11].

the sum of the light absorption coefficient $a$ and light scattering coefficient $b$, all in the unit $1 / \mathrm{m}$. The optically active components in natural waters are mainly colored dissolved organic matter (CDOM, also called yellow substances or gelbstoff), detritus (debris of plankton or sediments), and phytoplankton (microalgae). The optical properties $(a, b, c)$ of these generally decrease with wavelength, while (molecular) absorption by pure water increases with wavelength, as illustrated in Fig. 2.

\section{Light SOURCE}

\section{A. Green-Yellow Gap}

In the light attenuation spectrum of the Baltic Sea water (see Fig. 2), the minimum is in the green wavelength range of about 520-590 nm, which makes the use of green LEDs advantageous for optical communications [12], as the lowest attenuation is linked to the highest water transparency. Blue to green colored light (approximately 450-550-nm wavelength) is typically 


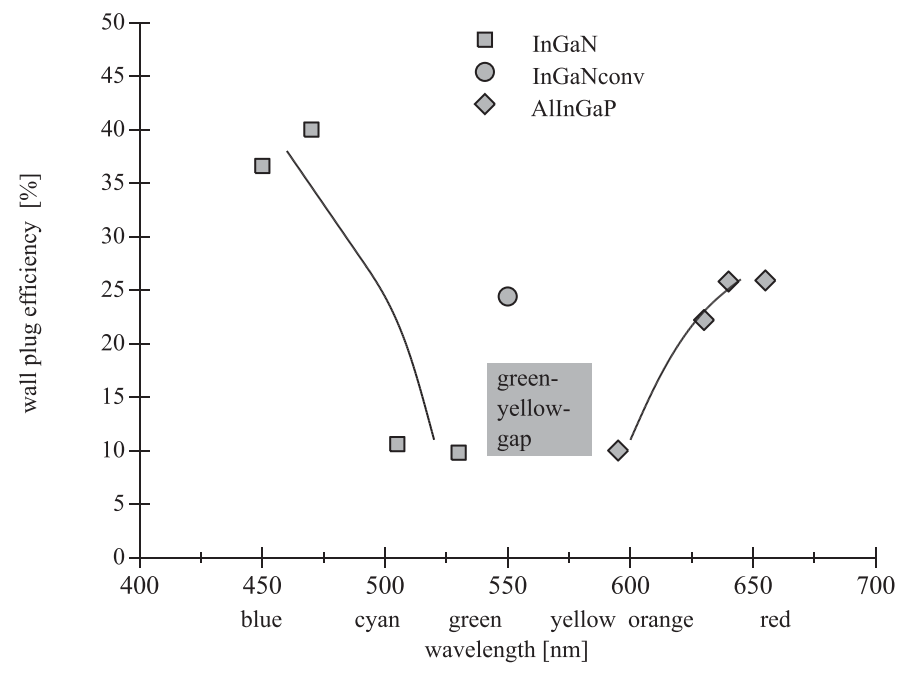

Fig. 3. LED wall-plug efficiency (also called radiant efficiency) of Luxeon $\mathrm{Z}$-series LEDs at 700-mA forward current. Values were calculated using intermediate binning from datasheet [14].

generated with indium gallium nitrite ( $\mathrm{InGaN})$ semiconductor LEDs. On the other hand, aluminum gallium indium phosphide (AlGaInP) semiconductor LEDs deliver yellow to red light (approximately $570-660 \mathrm{~nm}$ ). Although the market offers a huge variety of LEDs, in the green-yellow-orange color region only LEDs with a poor efficiency are currently available. This area is called the "green-yellow gap" of LEDs [13] (see Fig. 3), which is obviously just at the wavelengths of the lowest attenuation in coastal waters.

Announced a few years ago and still relatively rare in the market are converted green LEDs. They use highly efficient blue LEDs combined with a phosphor conversion layer to produce green light. These LEDs can potentially close the "green-yellow gap." A market survey of these commercially available LEDs reveals that the Luxeon Z-series is well suitable for testing. These off-the-shelf high-power single-color LEDs are well known for their high efficiency and are available in many colors including a converted green type, called "lime." One characteristic of this LED is a high spectral bandwidth (in relation to deep-blue and pure green LEDs), as illustrated in Fig. 4 and indicated by the large full-width half-maximum value in Table I. A minor power portion between approximately 600 and $700 \mathrm{~nm}$ (see Fig. 4) is in the range of higher attenuation (even higher than the attenuation of the blue regime) and, therefore, will be less effective for the received power of a detector.

\section{B. Light-Emitting Diode Speed}

A further disadvantage of converted green LEDs is the relatively slow phosphor-mediated conversion process, which limits the bandwidth to a few megahertz [15]-[17]. To determine the suitability of these converted LEDs for optical communication applications, the test setup shown in Fig. 5 was implemented. This setup consisted of a signal generator connected to a highspeed MOSFET switch to drive the LEDs with a square signal, and a fast photodetector linked to an oscilloscope to show the resulting peak shape.

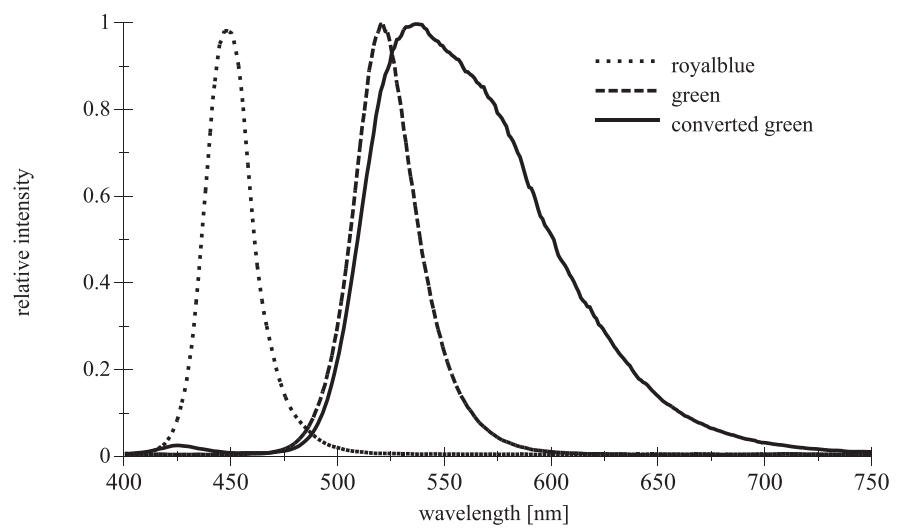

Fig. 4. Normalized light emission spectra of differently colored Luxeon Zseries LEDs (Lumileds Holding B.V., Amsterdam, The Netherlands) at 300-mA forward current measured using an USB2000 spectrometer (Ocean Optics, Inc., Dunedin, FL, USA).

TABLE I

Some Optical Properties of SeVERAl LEDS Used IN THIS StUdy

\begin{tabular}{lcccc}
\hline \hline $\begin{array}{l}\text { Manufacturer } \\
\text { Part no. }\end{array}$ & Color & $\begin{array}{c}\text { Peak } \\
\text { wavelength } \\
{[\mathrm{nm}]}\end{array}$ & $\begin{array}{c}\text { Spectral } \\
\text { half width } \\
{[\mathrm{nm}]}\end{array}$ & $\begin{array}{c}\text { Viewing } \\
\text { angle } \\
\text { [degree] }\end{array}$ \\
\hline Osram & & & & \\
LD W5SN & $\begin{array}{c}\text { deep-blue } \\
\text { blue }\end{array}$ & 449 & 25 & 120 \\
LB W5SN & green & 520 & 25 & 120 \\
LT W5SN & yellow & 597 & 33 & 120 \\
LY W5SN & & & 18 & 120 \\
Lumileds & royal blue & 450 & 20 & 125 \\
LXZ1-PR01 & green & 530 & 30 & 125 \\
LXZ1-PM01 & lime, & 550 & 100 & 125 \\
LXZ1-PX01 & conv. green & & & \\
& & & & \\
\hline \hline
\end{tabular}

[14], [20], [21]

Comparing the curves for royal blue and converted green in Fig. 6, the lowpass characteristic of the converted LED is visible. By using the rise time tr (in seconds) from $10 \%$ to $90 \%$ of the amplified photodetector signal, the approximate bandwidth BW (in hertz) can be determined as follows:

$$
\mathrm{BW} \approx \frac{0.35}{\operatorname{tr}} .
$$

In case of the tested converted green LED, a tr of $170 \mathrm{~ns}$ was identified, leading to a BW of slightly over $2 \mathrm{MHz}$. Based on this result, symbol rates in the order of a few megabits per second, depending on the signal-to-noise ratio (SNR), are theoretically achievable [18].

\section{Light-Emitting Diode Efficiency}

The radiant efficiency $\eta$ (also termed wall-plug efficiency) of an LED is defined as the ratio of emitted optical power $P_{\text {rad }}$ to the electrical input power $P_{\mathrm{el}}$, both in watts:

$$
\eta=\frac{P_{\mathrm{rad}}}{P_{\mathrm{el}}} .
$$

Wall-plug efficiency values for nine different LEDs are shown in Fig. 3. 


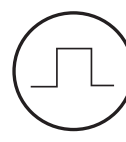

Rigol ${ }^{\circledR}$

DG 5072

function generator square signal

$\mathrm{f}=1 \mathrm{MHz}$

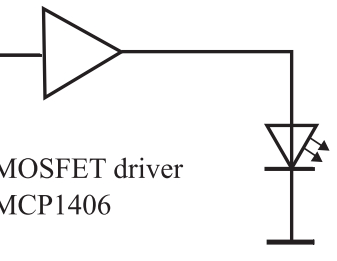

Lumileds $®$

Z Series LEDs royal blue, converted green $\mathrm{I}=700 \mathrm{~mA}$

Fig. 5. Setup used for testing the LED speed.

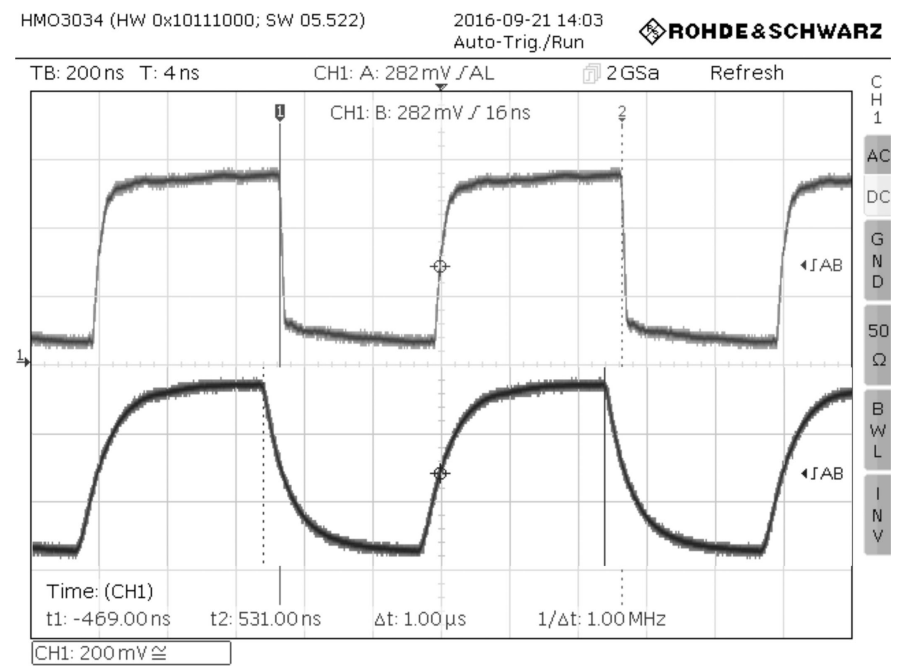

Fig. 6. Peak shape of Luxeon Z-series LEDs at $1 \mathrm{MHz}$. The plot shows overlayed oscilloscope screenshots, with the upper curve for royal blue LED and the lower curve for the converted green LED.

In datasheets for different LEDs, the radiated power in the visible spectral range is typically given for deep blue and deep red colors as a radiometric value (in watts). For wavelengths between deep blue and deep red, the photometric value (in lumen) is commonly documented and these photometric values are weighted by the photopic (daytime) human eyes sensitivity curve [19]. A simple conversion from photometric to radiometric units for a single wavelength can be performed using the luminous efficiency factor of $683 \mathrm{~lm} / \mathrm{W}$ at $555 \mathrm{~nm}$ and the photopic eye sensitivity function. However, LEDs are not monochromatic light sources. They show a nearly Gaussian-shaped spectral distribution, as pictured for royal blue and green in Fig. 4, thus a simple conversion is not accurate. To solve this problem, an integration of all contributing wavelengths is necessary, and this has been performed in 5-nm steps for the radiant power plotted in Fig. 3.

Instead of measuring the radiant power (in watts) directly, which would require expensive equipment such as an integrating Ulbricht sphere, the irradiance (in watts per square meter) at a constant distance is measured. For the comparison of LEDs, this simplification can be done, since the chosen LEDs show a radiant characteristic close to a Lambertian source, i.e., the
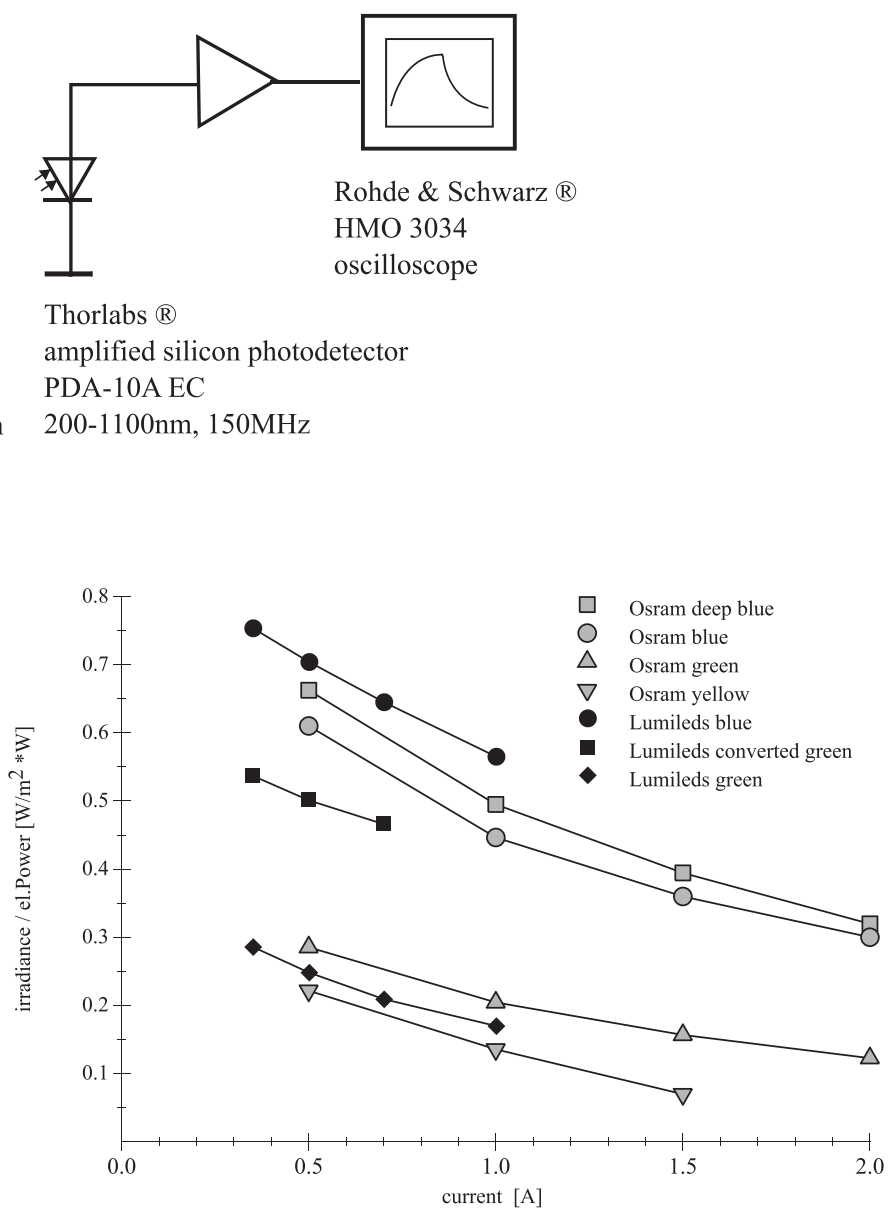

Fig. 7. Ratio of irradiance emitted per electrical power as a function of electrical current for different LED types and colors.

polar radiation pattern is almost circular and the total viewing angle at $50 \%$ intensity is approximately $120^{\circ}$ (see Table I). These measurements were conducted for different currents to examine changes in efficiency. These tests were performed in air, since the results at this stage do not require $\mathrm{u} / \mathrm{w}$ testing.

Fig. 7 shows that the blue LED has the highest ratio of irradiance to electrical input power, the converted green LED is less efficient, and the yellow and green LEDs show considerably lower efficiencies. Normalized to the values of the most efficient deep blue LED as 100\%, the converted green ones are offering efficiencies in the low $70 \%$, and the green ones only in the mid $30 \%$. The relation may slightly vary with current.

It is beneficial for the total efficiency to use more LEDs operated with lower currents, instead of forcing fewer LEDs using high currents. For further testing Luxeon Z-series LEDs in royal blue, green and converted green colors were selected.

\section{Reflector}

The radiation pattern of plain LEDs typically shows a wide and smoothly decreasing angular displacement, characteristic of a Lambertian source with an intensity of $50 \%$ at $60^{\circ}$ off axis. By installing a reflector, this can be changed toward a narrower 


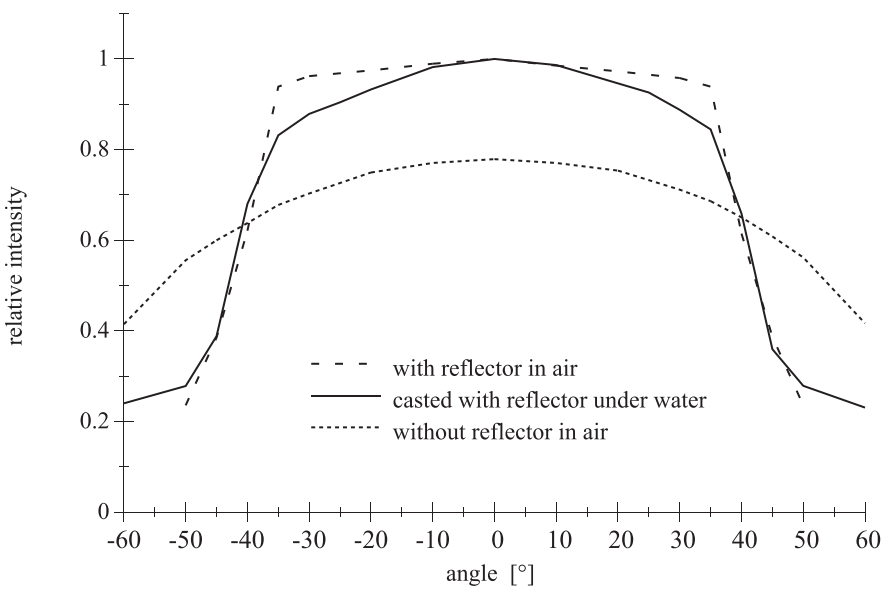

Fig. 8. Measured LED beam pattern with and without reflector in air and $\mathrm{u} / \mathrm{w}$. Tests were made on a goniometer bench. Four Luxeon Z-series LEDs tightly mounted on a metal-core printed circuit board were driven with $I=300 \mathrm{~mA}$. Intensity is gauged by an Extech LT 300 light meter with an aperture tube at $0.7-\mathrm{m}$ distance.

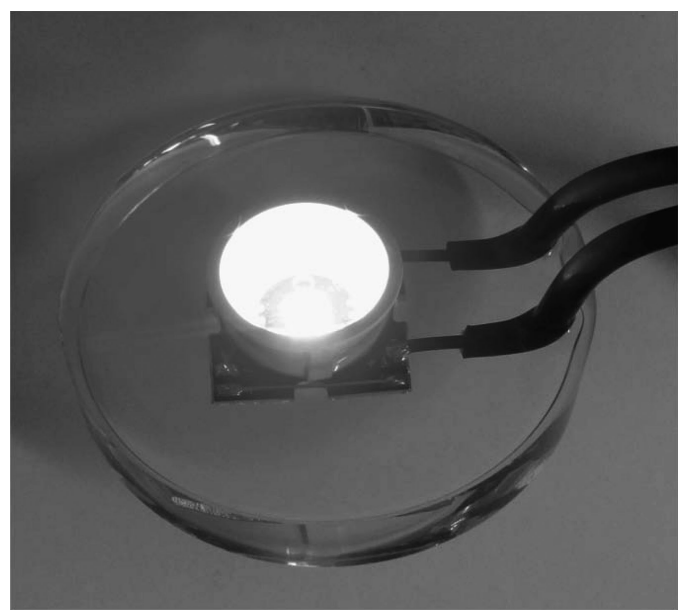

Fig. 9. Casted LED element with reflector for $\mathrm{u} / \mathrm{w}$ testing.

and more homogeneous pattern, but the irradiance in the center is increased at the cost of a reduced emission angle.

Fig. 8 demonstrates the measured relative spatial intensity of a quad LED element without a reflector in relation to the same LED element with reflector in air, and that of a casted element with reflector $\mathrm{u} / \mathrm{w}$. This LED element contains four Luxeon Z-series LEDs on a metal-core printed circuit board, bonded to a Carclo 10255 reflector and casted in highly transparent polyurethane, cf., Fig. 9. The flatness at the top of the angular pattern is slightly degraded (see Fig. 8) since the reflector base is flooded with a layer of polyurethane, which creates an extra transition in the optical path.

\section{PHOTODETECTOR}

\section{A. Sensitivity}

A benefit of using green or converted green instead of blue LEDs is the enhanced photosensitivity of PDs at longer visible wavelengths. The quantum efficiency (QE) for a given wavelength is the ratio of the generated photocurrent to the number

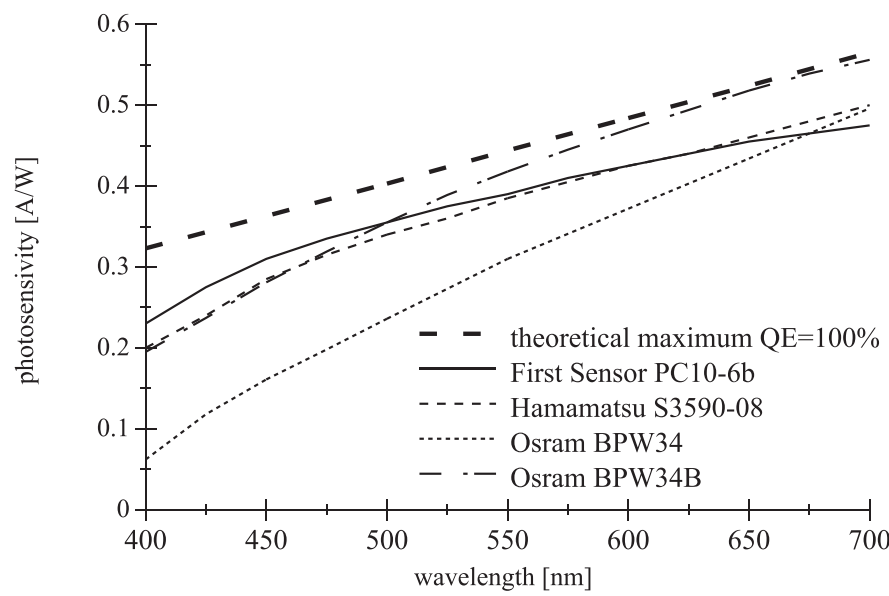

Fig. 10. Photosensitivity of standard and blue enhanced detectors.

TABLE II

PRoperties of ALl PHotodetectors Used IN THIS STUdy

\begin{tabular}{|c|c|c|c|c|c|}
\hline $\begin{array}{l}\text { Manufacturer } \\
\text { Part-No. }\end{array}$ & Type & $\begin{array}{c}\text { blue } \\
\text { enhanced }\end{array}$ & $\begin{array}{c}S_{b} \\
@ 450 \mathrm{~nm} \text { blue } \\
{[\mathrm{A} / \mathrm{W}]}\end{array}$ & $\begin{array}{c}S_{g} \\
@ 550 \mathrm{~nm} \text { green } \\
{[\mathrm{A} / \mathrm{W}]}\end{array}$ & $S_{g} / S$ \\
\hline \multicolumn{6}{|l|}{ Osram } \\
\hline BPW34 & Si PIN PD & no & 0.16 & 0.31 & 1.94 \\
\hline BPW34B & Si PIN PD & yes & 0.28 & 0.42 & 1.49 \\
\hline \multicolumn{6}{|l|}{ Advanced } \\
\hline \multicolumn{6}{|l|}{ Photonix } \\
\hline PDB-C156 & Si PIN PD & no & 0.1 & 0.22 & 2.2 \\
\hline PDB-C107 & Si PIN PD & yes & 0.19 & 0.31 & 1.63 \\
\hline \multicolumn{6}{|l|}{ Hamamatsu } \\
\hline S3590-08 & Si PIN PD & yes & 0.28 & 0.37 & 1.32 \\
\hline \multicolumn{6}{|l|}{ First Sensor } \\
\hline PC10-6b & Si PIN PD & yes & 0.32 & 0.39 & 1.22 \\
\hline AD1900-11 & Si APD & yes & $\begin{array}{c}28 \\
\text { gain }=100\end{array}$ & 34 & 1.21 \\
\hline
\end{tabular}

[23]-[28]; $S_{b}$ and $S_{g}$ are the sensitivities for blue and green. Silicon positive intrinsic negative photodiode (Si PIN PD). Silicon avalanche photodiode (Si APD).

of incident photons. In the visible range, the theoretical QE maximum is doubled from 400 to $800 \mathrm{~nm}$; see [22]. For the application in optical $\mathrm{u} / \mathrm{w}$ communication, blue enhanced devices are more suitable, as shown in Fig. 10. The sensitivity for blue and green lights of some common photodetectors is compared in Table II. We define the sensitivity ratio $\nu_{g b}$ of a PD as the photosensitivity $S_{g}$ (in ampere per watt) for green $(550 \mathrm{~nm})$ divided by the photosensitivity $S_{b}$ (in ampere per watt) for blue $(450 \mathrm{~nm})$ as follows:

$$
\nu_{g b}=\frac{S_{g}}{S_{b}} .
$$

In the best case, with a QE of $100 \%$ for both 450- and 550$\mathrm{nm}$ wavelengths, $\nu_{g b}$ achieves a value of 1.22 . This means the PD is $22 \%$ more sensitive for green as for blue. Even blue enhanced devices are often less close to the physical border $(\mathrm{QE}$ $=100 \%$ ) in the blue region as in the green, which increases $\nu_{g b}$. This ratio will be used later to compare blue and green colored systems. Of course such ratio can be formed for any other color or wavelength pairing, respectively. 


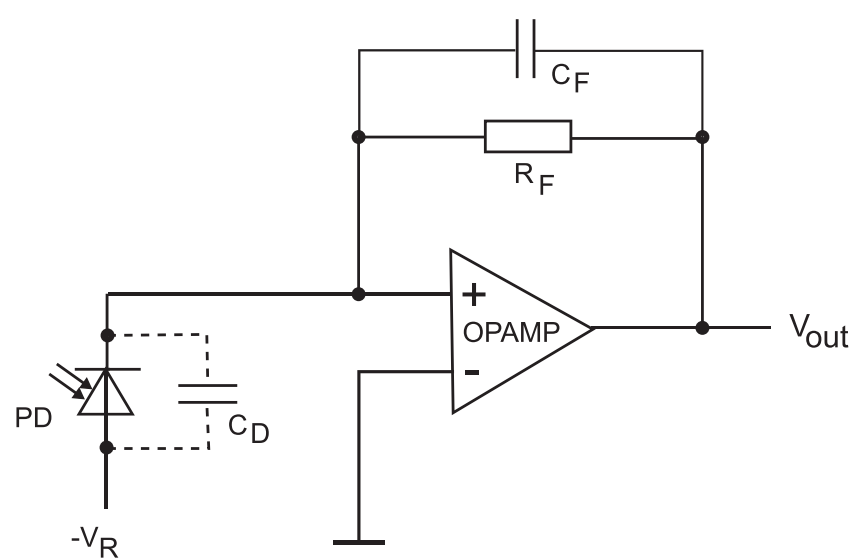

Fig. 11. Circuit of a basic TIA [32]. As an example, designing a TIA for an Osram BPW34B photodetector (as used later in the evaluation) by employing an Analog Devices AD8651 OPAMP for a target bandwidth of $1 \mathrm{MHz}$ and a maximal irradiance of $2 \mathrm{~W} / \mathrm{m}^{2}$ for $V_{\text {out }}=2 \mathrm{~V}$ output signal yields approximately $R_{F}=230 \mathrm{k} \Omega$ and $C_{F}=1 \mathrm{pF}$, when the PD is reversed biased with $V_{R}$ to reduce the junction capacitance $C_{D}$ to $15 \mathrm{pF}$.

\section{B. Amplification}

A p-i-n PD in the photoconductive mode usually generates a tiny current, which requires amplification and conversion into a voltage in a suitable range for further processing. For communication purposes, this will generally include a fast analogto-digital conversion with high resolution to offer a large dynamic range. The amplification is typically performed by a transimpedance amplifier (TIA), frequently a one-stage circuit consisting of a low-noise operational amplifier (OPAMP) with a high gain-bandwidth product and a resistor $R_{F}$ and a capacitor $C_{F}$ in the feedback loop, as illustrated in Fig. 11. In calculating a TIA for a particular PD, many variables have to be considered. The use of design tools for simulation is recommended [29]-[31].

\section{Noise Analysis}

According to the Shannon-Hartley theorem, the maximal achievable bitrate $R_{b}$ of a communication channel is expressed as

$$
R_{b}=\mathrm{BW} \cdot \log _{2}(1+\mathrm{SNR}) .
$$

In $\mathrm{u} / \mathrm{w}$ optical communications with LEDs and $\mathrm{p}-\mathrm{i}-\mathrm{n}$ PDs, the bandwidth limiting factors are typically the rise time of the LED, including driver at the transmitter side and the capacitance of the $\mathrm{PD}$ at the receiver side. The SNR is the most relevant parameter with respect to the achievable bitrate, i.e., the noise has to be kept as low as possible, since the strengthening of the signal would be power consuming and the signal would be attenuated quadratically and exponentially with the distance $r$; see (11). Ultimately, a decreased noise level will also boost the systems maximum reach.

The SNR equates as

$$
\mathrm{SNR}=\frac{i_{\text {signal }}^{2}}{i_{\text {noise }}^{2}}=\frac{\left(S \cdot P_{r}\right)^{2}}{\left(S \cdot \mathrm{NEP}_{\text {total }}\right)^{2}}=\frac{P_{r}^{2}}{\mathrm{NEP}_{\text {total }}^{2}}
$$

where $P_{r}$ is the received power at the PD and $S$ is the sensitivity, $i_{\text {signal }}$ is the average signal current, and $i_{\text {noise }}$ is the average noise current. The total noise equivalent power $\mathrm{NEP}_{\text {total }}$ has many contributors, such as shot noise, thermal noise, dark current noise, and preamplifier noise, and dependencies, such as sensitivity of PD, bandwidth, and temperature. Further sources can also be identified [3], [33], [34]. Typical dominant sources of noise are the shot noise, the thermal or Johnson noise in the feedback resistor $R_{F}$, and the PDs dark current noise, represented, respectively, as $\mathrm{NEP}_{\text {sigs }}, \mathrm{NEP}_{t R_{F}}$, and $\mathrm{NEP}_{i_{d}}$

$$
\begin{aligned}
\mathrm{NEP}_{\text {sigs }} & =\sqrt{\frac{2 \cdot q \cdot i_{\text {signal }} \cdot \mathrm{BW}}{S^{2}}} \\
\mathrm{NEP}_{t R_{F}} & =\sqrt{\frac{4 \cdot k \cdot T \cdot \mathrm{BW}}{R_{F} \cdot S^{2}}} \\
\mathrm{NEP}_{i_{d}} & =\sqrt{\frac{2 \cdot q \cdot i_{\mathrm{dark}} \cdot \mathrm{BW}}{S^{2}}} \\
\mathrm{NEP}_{\text {total }} & =\sqrt{\mathrm{NEP}_{\mathrm{sigs}}^{2}+\mathrm{NEP}_{t R_{F}}^{2}+\mathrm{NEP}_{i_{d}}^{2}} .
\end{aligned}
$$

Another important source is the noise of the amplifier. The corresponding values are the voltage noise density $e_{n}$ and the current noise density $i_{n}$, which can be found in the OPAMP datasheet. The calculation can be aided by design tools [30], [31]. Given noise voltages can be converted to currents through $R_{F}$ and currents via $S$ to NEP. The resulting $\mathrm{NEP}_{\mathrm{OPAMP}}$ has to be integrated into equation of the $\mathrm{NEP}_{\text {total }}$ (11). A simulation of the example in Fig. 11 yields a minimal detectable current, equal to the noise current $(\mathrm{SNR}=1)$, in the order of $1 \mathrm{nA}$. For further SNR calculations, this value will be used as $i_{\text {noise }}$.

\section{Optical Filter}

To reduce noise introduced by ambient light, optical filtering can be employed. Commonly used filters are colored glass or thin-film types with bandpass or shortpass characteristics [6]. Drawbacks of filters can be transmission losses and the dependence on the angle of incident. Care must be taken in designing a filter-photodetector combination for a wider FOV [35]. Optimal matching of filter curves to the LEDs spectra can be difficult to archive, since the standard assortment on the market is limited, or may require customized solutions. In our experiments, PDs without filters were used, to avoid another spectral-sensitive component in the setup. To eliminate daylight influence, the experiments were executed at dark night.

\section{Modulation Scheme}

A further issue of $\mathrm{u} / \mathrm{w}$ optical communications is the choice of the modulation scheme. Several feasibility studies compared the performance of these modulation techniques regarding bandwidth efficiency, power requirements, bit error performance, and implementation complexity [6], [9], [36]. Widely used is nonreturn-to-zero ON-OFF keying (NRZ-OOK) due to the simplicity in implementation. In consideration of the factors mentioned before, pulse position modulation (PPM) is recommended as a good compromise for applications like this. For further 
TABLE III

MODULATION SCHEME COMPARISON

\begin{tabular}{lcc}
\hline \hline Criteria & NRZ-OOK & 8-PPM \\
\hline $\begin{array}{l}\text { Bandwidth efficiency } \\
\text { Bandwidth BW in [hertz] } \\
\text { Bitrate } R_{b} \text { in [bits per second] }\end{array}$ & $R_{b}=\mathrm{BW}$ & $R_{b}=0.375 \cdot \mathrm{BW}$ \\
$\begin{array}{l}\text { Power efficiency } \\
\text { BER performance }\end{array}$ & low & high \\
$\begin{array}{l}\text { SNR in [decibel] } \\
\text { (for target BER of 1.0 e }-6)\end{array}$ & 13.5 & 14.0 \\
$\begin{array}{l}\text { (SNR per symbol) } \\
\text { Integration complexity }\end{array}$ & lowest & low \\
\hline \hline
\end{tabular}

[9], [36].

investigations and calculations, 8-PPM is used and compared with NRZ-OOK (see Table III).

The related bit error rate (BER) for NRZ-OOK is given as follows [37]:

$$
\text { BER }_{\text {NRZ-OOK }}=\frac{1}{2} \cdot \operatorname{erfc} \sqrt{0.5 \cdot \mathrm{SNR}} .
$$

Concerning the formula of the BER for 8-PPM, refer to equal energy orthogonal signals in [38]. Since the presented integral cannot be derived in closed form, the calculation requires some computational effort.

\section{EXPERIMENTAL PROCEDURE AND SETUP}

The irradiance $E$ (in watts per square meter) collected by the active area $A$ of the PD (in square meter) and the photosensitivity $S$ (in ampere per watt) determines the signal current $I$ (in amperes) as follows:

$$
I=E \cdot A \cdot S .
$$

If we assume that the LED is a point source with a specific emission angle, the irradiance $E$ seen by a detector decreases with distance $r$ following an inverse square law. The ratio of the irradiances at two distances $\left(E_{1}\right.$ and $\left.E_{2}\right)$ is proportional to the two distances $r_{1}$ and $r_{2}$ by

$$
\frac{E_{1}}{E_{2}}=\frac{r_{2}^{2}}{r_{1}^{2}} .
$$

Direct measurement of the received power is possible by a precision light meter containing an irradiance-detector head with a linear response in the visible range. This is advantageous when dealing with sources with various wavelengths, such as the three differently colored LEDs in the device under test (DUT). The sensor head is cosine corrected, thus the characteristic matches the angular sensitivity curve of a typical flat-substrate photodetector design, which is intended to be used for later setups. Furthermore, the waterproof version of the detector allows u/w measurements (Gigahertz-Optik P9710-4 optometer and RW3703 WQ detector head). Generally, a high absolute accuracy of irradiance measurements is hard to achieve and requires extensive calibration. Since our target is to undertake comparative measurements, a good linearity of the detector head is beneficial. The main factors for planning and executing $\mathrm{u} / \mathrm{w}$ tests in the

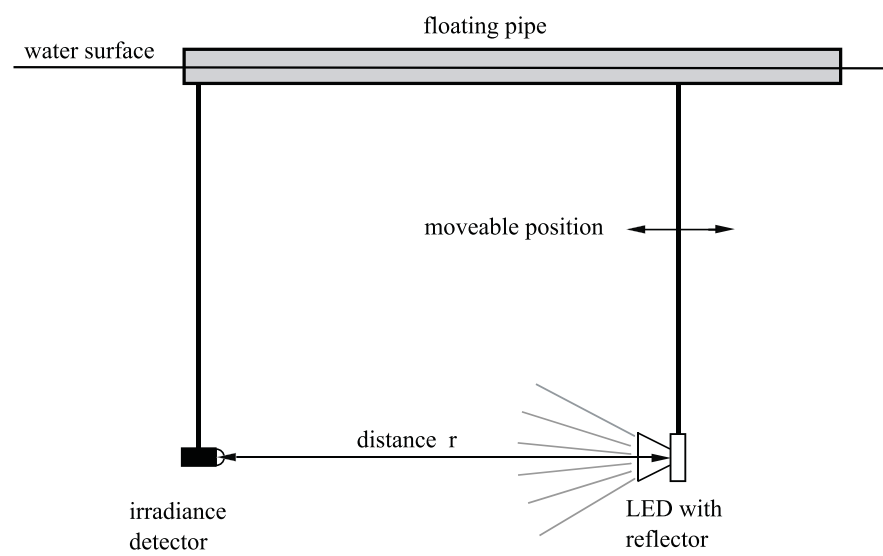

Fig. 12. Experimental setup with floating pipe for determining $k_{\mathrm{sys}}$.

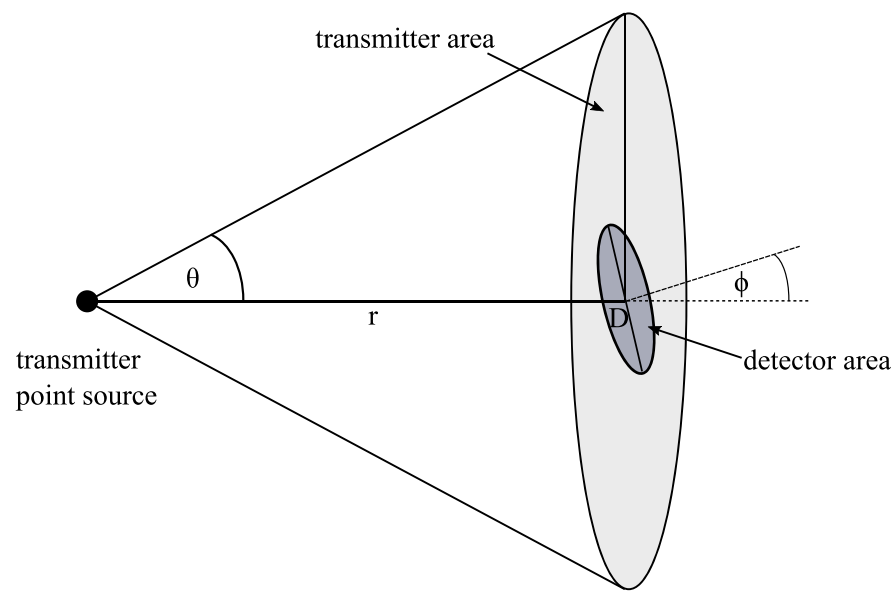

Fig. 13. Geometric layout of transmitter and receiver.

Baltic Sea are natural attenuation conditions in the seawater, no reflections from pool walls, and in our case easy access. To avoid or minimize the influence of ambient light, measurements were done at night. Although the water properties could not be changed actively, it is possible to repeat the measurements under different conditions, such as clearer or more turbid waters. It is important for later work that comprehensible parameters are recorded, e.g., measuring attenuation coefficient values by oceanographic instruments, such as a spectral transmissiometer [39]. A boundary condition for the test setup is no inclination between the LED(s) and the light sensor. This equipment should be in the line of sight and carefully aligned. Also, depth and distance of the LED(s) and detector should be kept at a certain ratio to minimize surface reflections. A sketch of the setup used in our experiment is shown in Fig. 12.

The received power of the system is affected by the properties of the transmitter, the medium, and the receiver. In [34], the effective received power $P_{r}$ is given by the formula

$$
P_{r}=\frac{P_{t} \cdot e^{-K \cdot r} \cdot D^{2} \cdot \cos \phi}{4 \cdot r^{2} \cdot \tan ^{2} \theta} .
$$

Using the conditions outlined in Fig. 13, $P_{r}$ is the received power and $P_{t}$ is the transmitted power (both in watts). The halfreflector opening angle is represented by $\theta$, the distance $r$ is in 
meters, and the attenuation coefficient $K$ has the unit $1 / \mathrm{m} . D$ stands for the detectors aperture diameter (in meters) and the angle $\phi$ represents the inclination with respect to the optical axis.

Calculations can be performed for each of the three different colored LEDs as DUT. The first uncertainty involves use of the correct water attenuation coefficient $c$ or $K$, or as stated before, probably a value in between. The second uncertainty is the value of the radiated power of the LED. The radiated power can either be directly measured using expensive instrumentation and possibly resulting in measurement error, or calculated based on datasheet curves of their relative spectral emission (subject to binning tolerances). Furthermore, an assumed ideal beam shape with uniform power density is probably not achievable, and must be taken into consideration.

On the other hand, field tests performed under real and constant conditions can deliver irradiance data at the receiver location(s), but it is important that the electrical power is kept constant. Let us denote the attenuation coefficient as $k_{\text {sys }}$, which is valid for the specific test setup, the water conditions, and the approximate peak wavelength of the light source (since the LED is not monochromatic) [6], [40].

An expansion of (15) with $\pi$ results in

$$
P_{r}=\frac{P_{t}}{\pi \cdot r^{2} \cdot \tan ^{2} \theta} \cdot e^{-K \cdot r} \cdot \frac{\pi \cdot D^{2} \cdot \cos \phi}{4} .
$$

Substituting $K$ by $k_{\text {sys }}$ and setting $\phi=0$ (no inclination) gives

$$
P_{r}=\frac{P_{t}}{\pi \cdot r^{2} \cdot \tan ^{2} \theta} \cdot e^{-k_{\mathrm{sys}} \cdot r} \cdot A_{\mathrm{det}} .
$$

The transmitter area term could also be represented by the area of a spherical crown yielding

$$
P_{r}=\frac{P_{t}}{2 \cdot \pi \cdot r^{2} \cdot(1-\cos \theta)} \cdot e^{-k_{\mathrm{sys}} \cdot r} \cdot A_{\mathrm{det}} .
$$

Using (18) will result in the same answer in (24). The irradiance at a distance $r$ can be expressed as

$$
E_{r}=\frac{P_{r}}{A_{\mathrm{det}}} .
$$

Solving for $P_{r}$ at two distances $r_{1}$ and $r_{2}$ gives

$$
\begin{aligned}
& P_{r 1}=E_{r 1} \cdot A_{\mathrm{det}} \\
& P_{r 2}=E_{r 2} \cdot A_{\mathrm{det}} .
\end{aligned}
$$

Substituting (20) into (18) and (21) into (18) results in

$$
\begin{aligned}
& E_{r 1}=\frac{P_{t}}{\pi \cdot r_{1}^{2} \cdot \tan ^{2} \theta} \cdot e^{-k_{\mathrm{sys}} \cdot r_{1}} \\
& E_{r 2}=\frac{P_{t}}{\pi \cdot r_{2}^{2} \cdot \tan ^{2} \theta} \cdot e^{-k_{\mathrm{sys}} \cdot r_{2}} .
\end{aligned}
$$

Forming the quotient of $E_{r 1}$ and $E_{r 2}$ leads to

$$
\frac{E_{r 1} \cdot r_{1}^{2}}{E_{r 2} \cdot r_{2}^{2}}=\frac{e^{-k_{\mathrm{sys}} \cdot r_{1}}}{e^{-k_{\mathrm{sys}} \cdot r_{2}}} .
$$

Finally, solving for $k_{\text {sys }}$ results in

$$
k_{\mathrm{sys}}=\frac{\ln \left(\frac{E_{r 1} \cdot r_{1}^{2}}{E_{r 2} \cdot r_{2}^{2}}\right)}{-r_{1}+r_{2}} .
$$

Hence, for every DUT LED with an individual wavelength, at least two irradiance measurements at different distances need to be undertaken. More measurements would be useful for confirming the persistence of the determined $k_{\text {sys }}$. Let us name these individual system coefficients $k_{\text {sysblue }}, k_{\text {sysgreen }}$, and $k_{\text {sysconvgreen }}$, respectively. By using $k_{\text {sys }}$, the ratio of two irradiances (e.g., the attenuation) at two distances $r_{1}$ and $r_{2}$ can be calculated as [41]

$$
\frac{E_{r 1}}{E_{r 2}}=e^{-k_{\mathrm{sys}} \cdot\left(r_{1}-r_{2}\right)} \cdot\left(\frac{r_{1}}{r_{2}}\right)^{2} .
$$

The next task is a comparison of the reach of two light sources fed with equal electrical power having the same optical characteristic (reflector), but different color-dependent radiant efficiencies, see also Section III-C. These properties of the DUT LEDs are leading to diverse values of the initial irradiance $E_{0}$ at the initial distance $r_{0}$. Different wavelengths are also causing individual attenuations. To fulfill this task, we want to determine the distance $r_{\mathrm{eq}}$, where the received power is the same for the two LEDs under investigation. This can be done efficiently by measuring the irradiance at the same distance $r_{0}$ for both DUTs, as illustrated in Fig. 14. A blue and a green LED pair was used, but the use of other color pairings such as blue and converted green would also be valid. Given $E=P / A$

$$
\begin{gathered}
P_{0 \text { blue }}=E_{0 \text { blue }} \cdot A_{0 \text { det }} \\
P_{0 \text { green }}=E_{0 \text { green }} \cdot A_{0 \text { det }} \cdot
\end{gathered}
$$

Using (17) for blue and for green and equating the received power $P_{r}$ results in

$$
\begin{aligned}
& \frac{E_{0 \text { blue }} \cdot A_{0 \text { det }}}{2 \cdot \pi \cdot r^{2} \cdot(1-\cos \theta)} \cdot e^{-k_{\text {sysblue }} \cdot r} \cdot A_{\text {det }} \\
= & \frac{E_{0 \text { green }} \cdot A_{0 \mathrm{det}}}{2 \cdot \pi \cdot r^{2} \cdot(1-\cos \theta)} \cdot e^{-k_{\text {sysgreen }} \cdot r} \cdot A_{\text {det }}
\end{aligned}
$$

and after simplification leads to

$$
E_{0 \text { blue }} \cdot e^{-k_{\text {sysblue }} \cdot r}=E_{0 \text { green }} \cdot e^{-k_{\text {sysgreen }} \cdot r} .
$$

Upon solving for $r$

$$
r=\frac{\ln \left(\frac{E_{0 \text { blue }}}{E_{0 \text { green }}}\right)}{\ln \left(\frac{e^{-k_{\text {sysgreen }}}}{e^{-k_{\text {sysblue }}}}\right)}
$$

yields

$$
r_{\mathrm{eq}}=r_{0}+r
$$




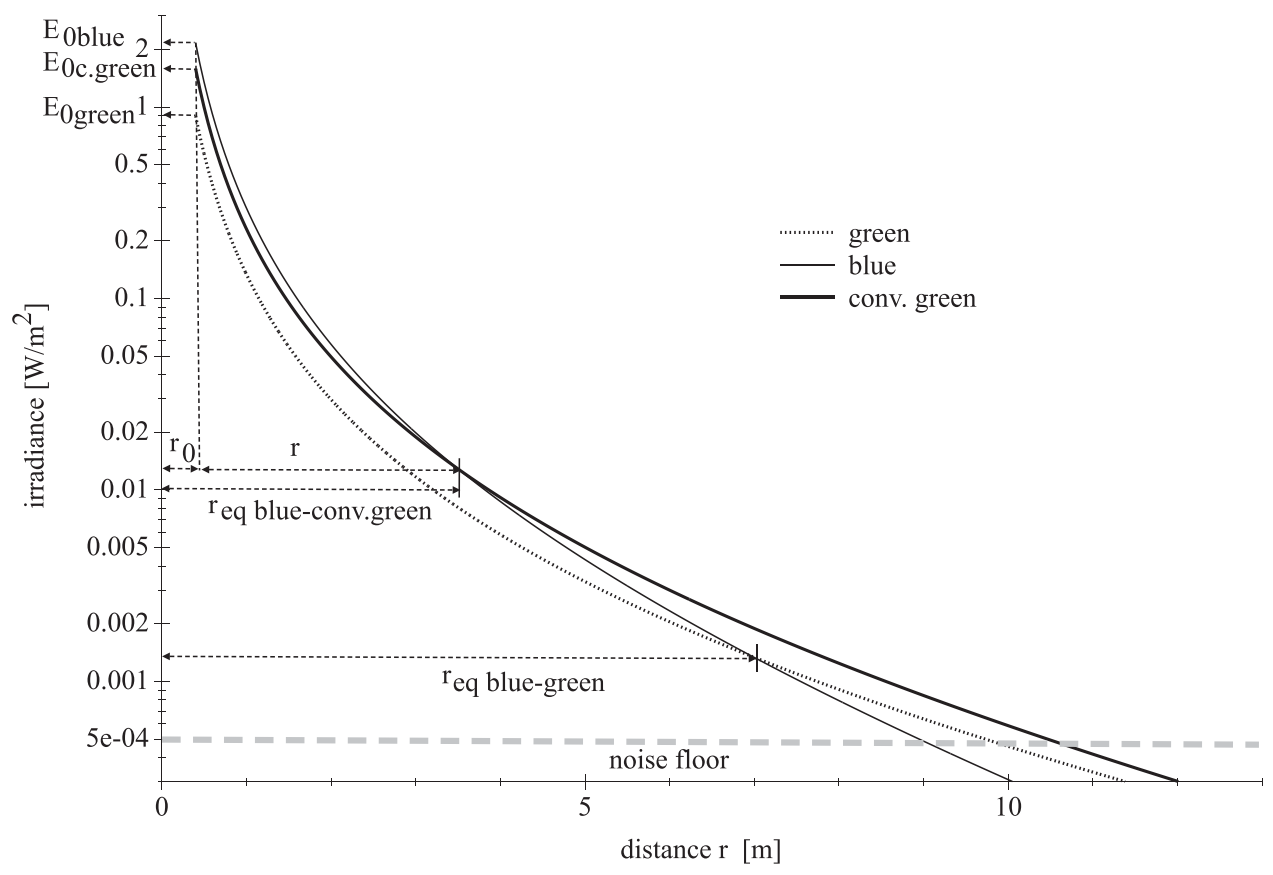

Fig. 14. Dependence of the irradiance from the distance between transmitter and receiver for different colored LEDs (see legend). Plot based on experimental data from August 18, 2016; see Table IV.

c.f., Fig. 14. The last step requires the substitution of $r$

$$
r_{\text {eq }}=r_{0}+\frac{\ln \left(\frac{E_{0 \text { blue }}}{E_{0 \text { green }}}\right)}{\ln \left(\frac{e^{-k_{\text {sysgreen }}}}{e^{-k_{\text {sysblue }}}}\right)} .
$$

To take the wavelength-dependent sensitivity of the PDs into account, the last equation can be extended by the sensitivity ratio $\nu_{g b}$ derived in (5). This will change the definition of $r_{\mathrm{eq}}$, being the distance of the same irradiance, into the distance of the same generated photocurrent $r_{\text {eqs }}$ as

$$
r_{\text {eqs }}=r_{0}+\frac{\ln \left(\frac{E_{0 \text { blue }}}{E_{0 \text { green }}} \cdot \frac{1}{\nu_{g b}}\right)}{\ln \left(\frac{e^{-k_{\text {sysgreen }}}}{e^{-k_{\text {sysblue }}}}\right)} .
$$

The determined values can be used to calculate the absolute values of power or irradiance at the PD at a distance $r_{\text {eq. }}$. Of course this equation can be modified to other color pairs. For example, blue and converted green with associated $\nu_{c g b}$.

The decision as to which LED color is beneficial can be guided by these calculations. For better understanding, let us consider two example pairs: blue/green versus blue/converted green LEDs, supported by the graphical presentation in Fig. 14. For the first blue/green pair, blue starts with a higher initial irradiance $E_{0 \text { blue }}$ than green $E_{0 \text { green }}$, but decreases faster due to higher attenuation. At larger distances beyond the calculated point of equal irradiance $r_{\text {eq blue-green }}$, the signal of a green LED would be stronger.

Concerning the second blue/converted green pair, a converted green LED has a relatively high initial power value $E_{0 c \text {.green }}$ too, but the attenuation of converted green is only slightly higher than for green and much less as for blue. This leads into an intersection with blue at a comparatively shorter distance at $r_{\text {eq blue-conv.green }}$

Now the noise floor has to be taken into consideration. It represents a practical lower limit of a detectable signal, see also Sections IV-B and IV-C. Since we seek for a maximization of the signal, hence SNR, the curve intersecting the noise floor at the largest distance is proposed to deliver the strongest signal. For short distances, another color may overtake, in this example blue up to $r_{\text {eq blue-conv.green }}$, but this has less importance because the SNR is high at these short distances anyway.

The next step is to develop a calculation of the SNR, one of the most important parameter for communications. To integrate, the PDs wavelength-dependent sensitivity requires changing the view from irradiances to currents [42]. Combining (3), (19), and (26) leads to the SNR at distance $r_{1}$

$$
\mathrm{SNR}=\frac{\left(S \cdot A_{\mathrm{det}} \cdot E_{0} \cdot \frac{r_{0}^{2}}{r_{1}^{2}} \cdot e^{-k_{\mathrm{sys}}\left(r_{1}-r_{0}\right)}\right)^{2}}{\left(i_{\text {noise }}\right)^{2}} .
$$

Based on the measurements of the initial values $E_{0}$ and $r_{0}$, the determined $k_{\mathrm{sys}}$ for the investigated colors and the identified $i_{\text {noise }}$, we are now in the position to calculate the SNR and thereafter to compare the BER of different modulation schemes at a certain distance. A practical way to conduct this evaluation is to use a spreadsheet program.

\section{EXPERIMENTAL RESULTS}

To determine the individual $k_{\mathrm{sys}}$ of the three different colored LEDs, five experiments were performed in summer 2016 in the Eckernfoerde Bight at coordinates $54^{\circ} 28.490^{\prime} \mathrm{N}$ and $9^{\circ} 50.803^{\prime}$ E. The intention was to evaluate the proposed method under 
TABLE IV

EXPERIMENTAL RESULTS

\begin{tabular}{|c|c|c|c|c|c|}
\hline & July 23 & August 18 & August 26 & September 6 & September 28 \\
\hline Visibility, subjective in meters & 2 & 4 & 4 & 3 & 2 \\
\hline Temperature of water in degree celcius & 20 & 19 & 19 & 18 & 18 \\
\hline \multicolumn{6}{|l|}{$k_{\mathrm{sys}}$ in $1 / \mathrm{m}$} \\
\hline For blue & 0.311 & 0.252 & 0.198 & 0.224 & 0.434 \\
\hline For green & - & 0.119 & 0.106 & 0.132 & 0.195 \\
\hline For converted green & 0.164 & 0.157 & 0.142 & 0.182 & 0.237 \\
\hline \multicolumn{6}{|l|}{$r_{\mathrm{eq}}$ in meters } \\
\hline For blue $(450 \mathrm{~nm})$-converted green $(550 \mathrm{~nm})$ & 2.52 & 3.67 & 6.41 & 9.40 & 2.24 \\
\hline \multicolumn{6}{|l|}{$r_{\mathrm{eq}}$ in meters } \\
\hline For blue $(450 \mathrm{~nm})$-green $(525 \mathrm{~nm})$ & - & 6.99 & 10.4 & 10.7 & 4.48 \\
\hline \multicolumn{6}{|l|}{$r_{\text {eqs }}$ in meters with $\nu_{g b}=1.22$} \\
\hline \multicolumn{6}{|l|}{$r_{\text {eqs }}$ in meters with $\nu_{g b}=1.17$} \\
\hline For blue $(450 \mathrm{~nm})$-green $(525 \mathrm{~nm})$ & - & 5.81 & 8.66 & 9.00 & 3.82 \\
\hline
\end{tabular}

Data of Summer 2016 in the Eckernfoerde Bight at $54^{\circ} 28.490^{\prime} \mathrm{N}$ and $9^{\circ} 50.803^{\prime} \mathrm{E}$.

different optical water conditions and to improve the setup. The LEDs were driven with the same constant current during the experiments and related forward voltages were recorded. To achieve equal electrical input power values for following calculations, corrections were applied afterward to the measured irradiance values assuming that the radiant efficiency of the LED is linear in this narrow range. This is essential with respect to the comparison of measured irradiance values. As the spectral sensitivity of the irradiance detector head is known, minor corrections for the individual wavelengths were applied. No additional filters in the optical path were used. Since the irradiance decrease is dominated by the geometric loss, care was taken in selecting the two distances $r_{1}$ and $r_{2}$. If the distance between $r_{1}$ and $r_{2}$ is too small, the accuracy of the determination of this distance is insufficient. If the distance is too large, irradiance levels become too low for an accurate determination of the difference in power. In this experiment, an LED of power $3 \mathrm{~W}$ and distances of 0.4-0.8 $\mathrm{m}$ were used for $r_{1}$, and 1.3-2.2 $\mathrm{m}$ for $r_{2}$. Since the measurements were conducted under natural conditions, signal variations due to scattering from particles drifting in the current were averaged by using a long integration time of $5 \mathrm{~s}$. Although the measurements were performed at night, ambient light values and meter offsets were taken into account. Table IV and Fig. 15 show the results.

During two days of relatively clear water conditions, an u/w spectrophotometer (AC-S, WetLABS, Philomath, OR, USA) was used for measuring in situ optical properties. This allowed a comparison of the determined $k_{\text {sys }}$ with the attenuation, absorption, and scattering coefficients measured by the AC-S equipment [43], as presented in Fig. 16. On both days, the determined values for $k_{\text {sys }}$ were close to absorption coefficient values, which indicates negligible loss due to scattering at these short distances [6].

When comparing the values of $k_{\mathrm{sys}}$ for green and converted green LEDs in Figs. 15 and 16, experiments with converted green show higher $k_{\text {sys }}$ values. This effect is related to a significant part of the spectral bandwidth above $580 \mathrm{~nm}$ (see Fig. 4). As presented in Table IV, the converted green LEDs have sig-

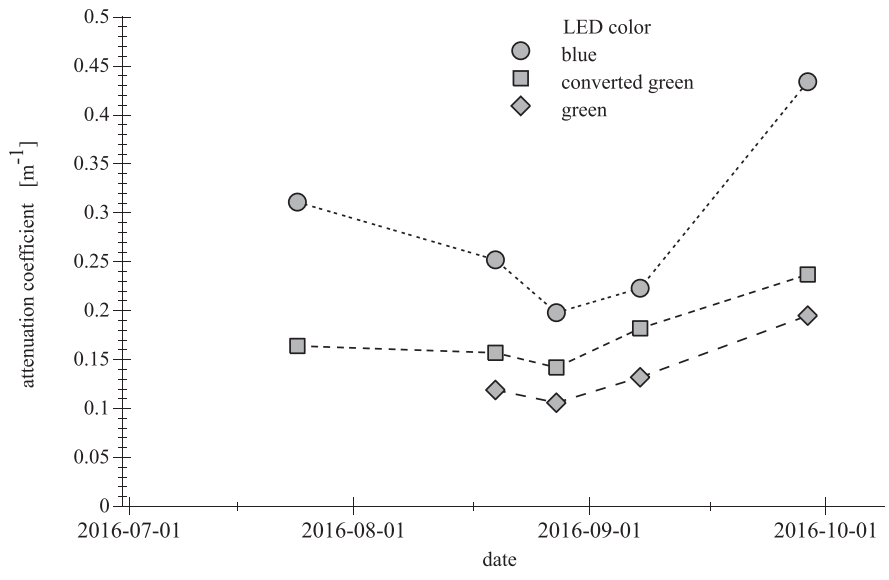

Fig. 15. Determined attenuation coefficient $k_{\mathrm{sys}}$, based on measurements in the Baltic Sea in summer 2016.

nificant shorter $r_{\text {eqs }}$ values compared to the green LEDs, thus they can be a substitution for blue or green LEDs in coastal waters.

The found water conditions in the Baltic Sea during the field tests were relatively clear, in the range of Jerlov water type 3C. Referring to Fig. 1, more turbid coastal waters such as 5C-9C, where the attenuation minimum shifts toward higher wavelengths, would further benefit the results of the converted green LED.

Effects of power variations and noise floor levels can be identified in Fig. 17. Looking at the one extreme of very small power and a lifted noise floor, the result is that at short distances blue can be the better choice. Contrariwise with very high power and a lowered noise floor, the outcome is a long distance and green can be the better choice here. It can also be distinguished that decreasing the noise floor level can have a similar effect as increasing the power. The impact on the important communication parameter BER is shown for two modulation schemes with varying conditions of four experimental days in Figs. 18 and 19. Keeping the BER constant, the converted green LEDs are archiving the largest distances. Similarly, keeping the dis- 


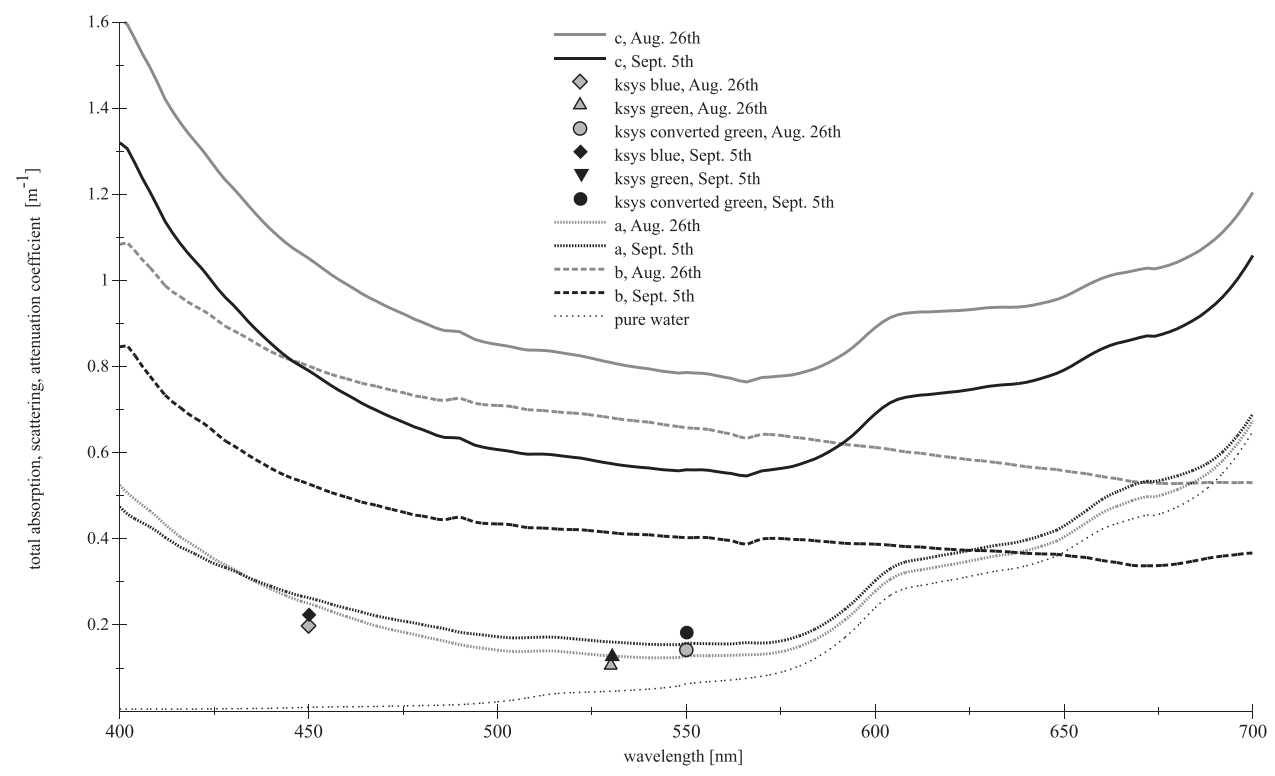

Fig. 16. Measurements in the Baltic Sea showing spectral optical properties and the determined $k_{\text {sys }}$ values on August 26 and September 5, 2016. ACS data including correction for pure water attenuation and scatter [43]. Note that $\mathrm{a}=$ absorption; $\mathrm{b}=$ scattering; and $\mathrm{c}=$ attenuation.

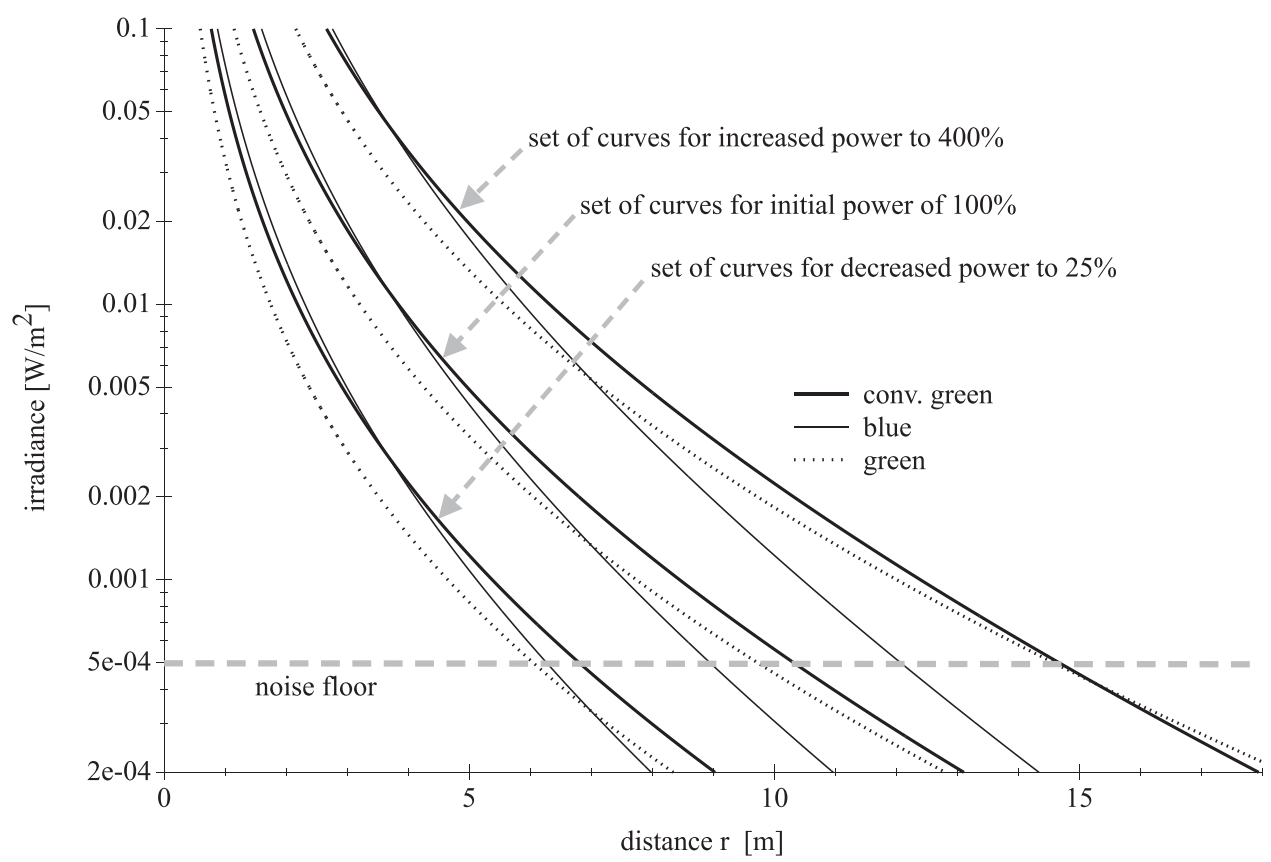

Fig. 17. Graphics is showing the influence of power variations to the irradiance and achievable distance between LEDs and PD for a certain noise floor level for different colors (see legend). Projected to a spherical radiation the initial example (middle set of curves) corresponds to approximately $25 \mathrm{~W}$ of electrical power. So the three sets of curves are located in a practical quite possible range. Plot based on experimental data from August 18, 2016; see Table IV.

tances constant results in the lowest BER for the use of converted green. The decision which modulation scheme to use in this case is up to the preference of maximizing the distance and the energy efficiency or the bitrate; cf., Table III.

\section{CONCLUSION AND Future WORK}

In the Baltic Sea and other waters with similar optical properties, converted green LEDs can outperform the slightly higher energy-efficient blue LEDs due to the higher light transmission at green wavelengths. This advantage of converted green LEDs is even better in more turbid waters. However, a disadvantage of the converted green LEDs is the limited speed of the phosphor conversion process. Taking into consideration that even blue enhanced silicon photodetectors are typically $20 \%$ more sensitive for green as for blue light, the choice of converted green LEDs for optical $\mathrm{u} / \mathrm{w}$ communication systems up to several megabits per second is confirmed.

It would be desirable for $\mathrm{u} / \mathrm{w}$ communications if the LED market would offer faster color converted LEDs with lower spectral 


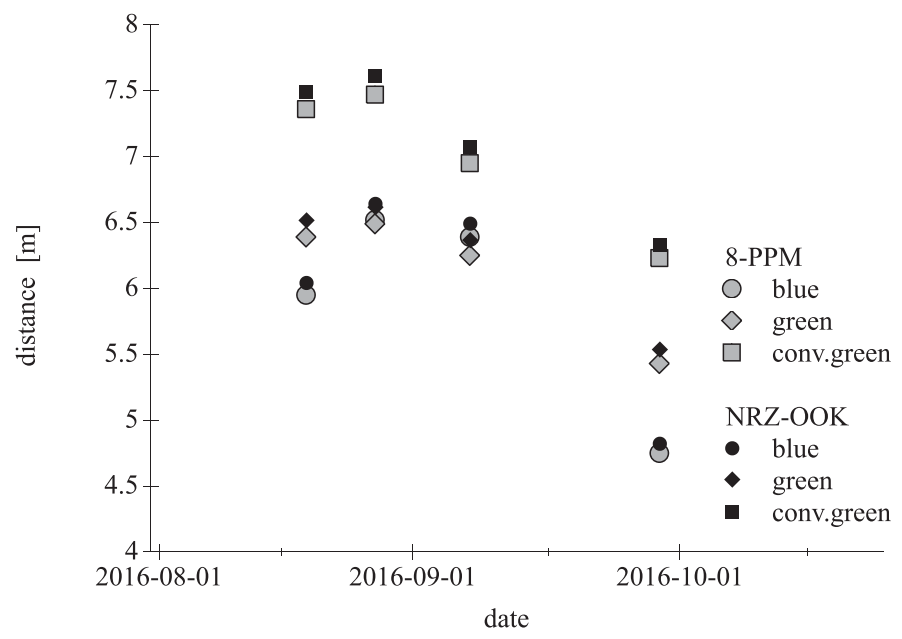

Fig. 18. Comparison of the calculated attainable distance between LED and PD for two modulation schemes and three colors, based on measurements at four days under varying water parameters $k_{\text {sys }}$ in the Baltic Sea in summer 2016. Boundary conditions are: LEDs driven with same electrical power, target BER of $1.0 \cdot \mathrm{e}-6, i_{\text {noise }}$ of $1 \mathrm{nA}$, Osram BPW34B photodetector and Lumileds $\mathrm{Z}$ series LEDs.

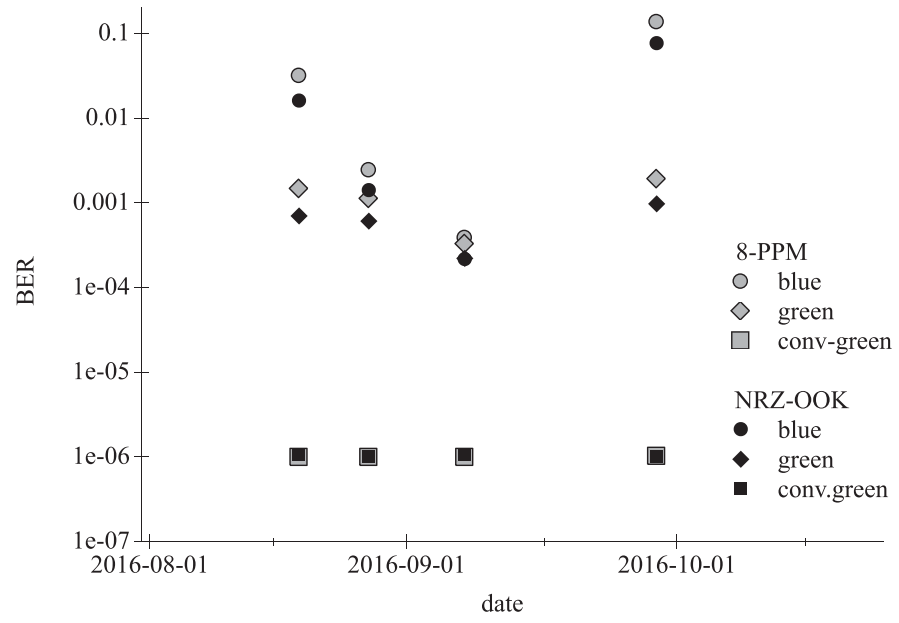

Fig. 19. Comparison of the calculated BER for two modulation schemes and three LED colors at the same distance between LED and PD, based on measurements at four days under varying water parameters $k_{\mathrm{sys}}$ in the Baltic Sea in summer 2016. Boundary conditions are: LEDs driven with same electrical power, target BER of $1.0 \cdot \mathrm{e}-6$ for converted green LED, $i_{\text {noise }}$ of $1 \mathrm{nA}$, Osram BPW34B Photodetector and Lumileds Z series LEDs. The values of the corresponding distances are: August 18, $7.36 \mathrm{~m}$ (8-PPM) and $7.49 \mathrm{~m}$ (NRZOOK); August 26, $7.47 \mathrm{~m}$ (8-PPM) and $7.61 \mathrm{~m}$ (NRZ-OOK); September 6, $6.95 \mathrm{~m}$ (8-PPM) and $7.07 \mathrm{~m}$ (NRZ-OOK); September 28, $6.23 \mathrm{~m}$ (8-PPM) and $6.33 \mathrm{~m}$ (NRZ-OOK). These values are also extractable from Fig. 18.

bandwidth and widen the choice of wavelengths. A matter of importance for the design of systems with low energy consumption is beside the use of efficient LEDs, the performance of the photodetector, and a low noise level. Since many variables and their relation to each other play a role, a reliable prediction without calculation or simulation can be difficult.

The general scope of the future work is the modeling of the $\mathrm{u} / \mathrm{w}$ optical channel for communication purposes. Since many influencing factors can be identified on the path from the light source to the detector, these factors should be considered in simulation software. The light source is the first of three main blocks, including the LED type, the power, a reflector, a window, and the geometrical setup. The second block contains the water parameters, depth, ambient light, and the distance. The third comprises the detector, the window, a filter, the geometrical setup, and a basic amplifier. The plan is to provide some standard off-the-shelf parts as predefined LEDs and photodetectors, as well as predefined water classes and some basic ambient light scenarios. The outcome will be the SNR and the bandwidth for the selected parts under chosen conditions. Based on these values, the performance of different modulation schemes can be distinguished. The objective is to support the choice of parts and to provide an opportunity to estimate the systems behavior under different conditions. The aim is to ease the way toward viable, robust, powerful, and efficient $\mathrm{u} / \mathrm{w}$ optical communication systems.

\section{ACKNOWLEDGMENT}

The authors would like to thank H. Burmester for preparing the Wetlabs ACS transmissiometer and C. Utschig for data analysis, both from Helmholtz Zentrum Geesthacht.

\section{REFERENCES}

[1] F. Menna, E. Nocerino, F. Fassi, and F. Remondino, "Geometric and optic characterization of a hemispherical dome port for optical underwater photogrammetry," Sensors, vol. 16, no. 48, pp. 1-21, 2016.

[2] J. Simpson, B. L. Hughes, and J. F. Muth, "Smart transmitters and receivers for underwater free-space optical communication," IEEE J. Sel. Areas Commun., vol. 30, no. 5, pp. 964-974, Jun. 2012.

[3] J. W. Giles and I. N. Bankman, "Underwater optical communications part 2: Basic design considerations," in Proc. IEEE Mil. Commun. Conf., 2005, pp. 1700-1705.

[4] J. H. Smart, "Underwater optical communications part 1: Variability of water optical parameters," in Proc. IEEE Mil. Commun. Conf., 2005, pp. $1140-1146$.

[5] C. D. Mobley et al., Ocean Optics Web Book, Oct. 2016. [Online]. Available: www.oceanopticbook.info

[6] S. Arnon, J. R. Barry, G. K. Karagiannidis, R. Schober, and M. Uysal, Ed., Advanced Wireless Optical Communication Systems. Cambridge U.K.: Cambridge Univ. Press, 2012, ch. 9, pp. 201-272, 2012.

[7] M. G. Solonenko and C. D. Mobley, "Inherent optical properties of Jerlov Water types," Appl. Opt., vol. 54, no. 17, pp. 5392-5401, 2015.

[8] E. Rosenkrantz and S. Arnon, "Optimum LED wavelength for underwater optical wireless communication at turbid water," Proc. SPIE, vol. 9224, no. 4, pp. 922413-1-922413-6, Oct. 2014.

[9] S. Meihong, Y. Xinsheng, and Z. Fengli, "The evaluation of modulation techniques for underwater wireless optical communications," in Proc. IEEE Int. Conf. Commun. Softw. Netw., Feb. 2009, pp. 138-142.

[10] E. Aas, N. K. Højerslev, J. Høkedal, and K. Sørensen, "Optical water types of the Nordic Seas and adjacent areas," Oceanologia, vol. 55 , no. 2 , pp. 471-482, 2013.

[11] M. Edman, "Modelling light attenuation in the Baltic Sea," Earth Science Centre, Göteborg Univ., Gothenburg, Sweden, Project Rep. B486, pp. 1-36, 2006.

[12] I. Levin, M. Darecki, S. Sagan, and T. Radomyslskaya, "Relationships between inherent optical properties in the Baltic Sea for application to the underwater imaging problem," Oceanologia, vol. 55, no. 1, pp. 11-26, 2013.

[13] R. Mueller-Mach et al., "All nitride monochromatic amber-emitting phosphor-converted light emitting diodes," Physica Status Solidi Rapid Res. Lett., vol. 3, no. 7/8, pp. 215-217, 2009.

[14] "LUXEON Z colors product datasheet," Lumileds Holding B.V., Amsterdam, The Netherlands, DS105, 2015.

[15] D. O'Brien et al., "Visible light communications: Challenges and possibilities," in Proc. IEEE Int. Symp. Pers., Indoor Mobile Radio Commun., Sep. 2008, pp. 1-5. 
[16] I. Dursun et al., "Perovskite nanocrystals as a color converter for visible light communication," ACS Photon., vol. 2016, no. 3, pp. 1150-1156, May 2016.

[17] H. Chun et al., "Visible light communication using a blue GaN uLED and flourescent polymer colour converter," IEEE Photon. Technol. Lett., vol. 26, no. 20, pp. 2035-2038, Aug. 2014.

[18] D. Anguita, D. Brizzolara, and Q. Hu, "Optical wireless underwater communication for AUV: Preliminary simulation and experimental results," in Proc. IEEE OCEANS Conf., Jun. 2011, pp. 1-5.

[19] "CIE $19882^{\circ}$ spectral luminous efficiency function for photopic vision," CIE 086-1990, 1990.

[20] "Platinum dragon LDW5SN LBW5SN LTW5SN," OSRAM Opto Semiconductor GmbH, Regensburg, Germany, 2010.

[21] "Platinum dragon LRW5SN LAW5SN LYW5SN," OSRAM Opto Semiconductor GmbH, Regensburg, Germany, 2008.

[22] "Si photodiodes," in Opto Semiconductor Handbook. Hamamatsu City, Japan: Hamamatsu Photonics K.K., 2014, ch. 2.

[23] "Silicon PIN photodiode BPW34 BPW34S BPW34SR," OSRAM Opto Semiconductor GmbH, Regensburg, Germany, 2007.

[24] "Blue enhanced photoconductive silicon photodiode PDB-C107," Advanced Photonix, Inc., Ann Arbor, MI, USA.

[25] "Plastic photodiode packages with leads PDB-C156," Advanced Photonix, Inc., Ann Arbor, MI, USA.

[26] "Si PIN photodiode S3590-08 /-09/-18/-19," Hamamatsu Photonics K.K., Hamamatsu City, Japan, 2012.

[27] "First sensor PIN PD data sheet PC10-6b TO," First Sensor AG, Berlin, Germany, 2013.

[28] "First sensor APD data sheet AD1900-11 TO," First Sensor AG, Berlin, Germany, 2011.

[29] G. Graeme, Photodiode Amplifiers. New York, NY, USA: McGraw-Hill, 1995, ch. 5, pp. 87-106.

[30] "Photodiode circuit design wizard," Analog Devices, Inc., Norwood, MA, USA. [Online]. Available: www.analog.com/designtools/en/photodiode, Accessed on: Aug.1, 2017.

[31] JavaScience, “Transimpedance amplifiers," JavaScience Consulting. [Online]. Available: www.jensign.com/transimpedance/index.html, Accessed on: Aug. 1, 2015.

[32] M. Doniec, M. Angermann, and D. Rus, "An end-to-end signal strength model for underwater optical communications," IEEE J. Ocean. Eng., vol. 38, no. 4, pp. 743-757, Oct. 2013.

[33] V. Mackowiak et al., "NEP noise equivalent power," Thorlabs, Inc., Newton, NJ, USA, White Paper, 2015.

[34] M. Lanzagorta, Underwater Communications. San Rafael, CA, USA: Morgan \& Claypool, 2012, pp. 55-68.

[35] J. M. Kahn and J. R. Barry, "Wireless infrared communications," Proc. IEEE, vol. 85, no. 2, pp. 265-298, Feb. 1997

[36] C. Gabriel et al., "Investigation of suitable modulation techniques for underwater wireless optical communication," in Proc. IEEE Int. Workshop Opt. Wireless Commun., 2012, pp. 1-3.

[37] R. M. Gagliardi and S. Karp, Optical Communications. Hoboken, NJ, USA: Wiley, 1995, pp. 181-191.

[38] J. M. Proakis, Digital Communications. New York, NY, USA: McGrawHill, 1995, pp. 260-264.

[39] J. R. Potter, M. B. Porter, and J. C. Preisig, "UComms: A conference and workshop on underwater communications, channel modeling, and validation," IEEE J. Ocean. Eng., vol. 38, no. 4, pp. 603-613, Oct. 2013.

[40] B. M. Cochenour, L. J. Mullen, and A. E. Laux, "Characterization of the beam-spread function for underwater wireless optical communications links," IEEE J. Ocean. Eng., vol. 33, no. 4, pp. 513-521, Oct. 2008.

[41] D. Anguita, D. Brizzolara, and G. Parodi, "Prospects and problems of optical diffuse wireless communication for underwater wireless sensor networks (UWSNs)," in Wireless Sensor Networks: Application-Centric Design. Rijeka, Croatia: InTech, 2010, pp. 275-300.

[42] I. Vasilescu et al., "Data collection, storage, and retrieval with an underwater sensor network," in Proc. ACM 3rd Int. Conf. Embedded Networked Sensor Syst., 2005, pp. 154-164.

[43] R. Röttgers, D. McKee, and S. B. Wozniak, "Evaluation of scatter corrections for ac-9 absorption measurements in coastal waters," Methods Oceanogr., vol. 7, pp. 21-39, 2013.

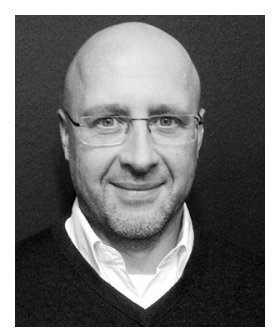

Jan Sticklus (M'16) received the Dipl.Ing. degree in electrical engineering from Kiel University of Applied Sciences, Kiel, Germany, in 1993. He is currently working toward the Ph.D. degree at the Faculty of Engineering, University of Kiel, Kiel, Germany.

Since then, he has been an Engineer at the GEOMAR Helmholtz Centre for Ocean Research Kiel. From 2007 to 2014, he was a member of the GEOMAR AUV team, working with autonomous underwater vehicles in worldwide deep sea operations. His research interests include optical underwater communications as well as LED and pressure neutral technology.

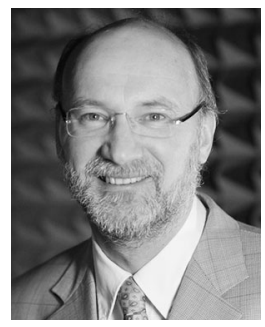

Peter Adam Hoeher (F'14) received the M.Sc. degree in electrical engineering from RWTH Aachen University, Aachen, Germany, in 1986 and the Ph.D. degree in electrical engineering from the University of Kaiserslautern, Kaiserslautern, Germany, in 1990.

From October 1986 to September 1998, he was with the German Aerospace Center (DLR), Oberpfaffenhofen, Germany. Between December 1991 and November 1992, he was on leave from AT\&T Bell Laboratories, Murray Hill, NJ, USA. In October 1998, he joined the University of Kiel, Kiel, Germany, where he is now a Full Professor of Electrical and Information Engineering. His research interests include the general area of communication theory and applied information theory with applications in wireless radio communications, underwater communications, wireless optical communications, and molecular communications.

Dr. Hoeher received the Hugo-Denkmeier-Award'90, the ITG Award'07, and several best paper awards. Between 1999 and 2006, he served as an Associated Editor for the IEEE TRANSACTIONS ON COMMUNICATIONS. In 2014, he became an IEEE Fellow for his contributions to decoding and detection that include reliability information.

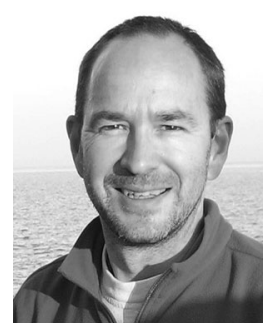

Rüdiger Röttgers received M.Sc. and Ph.D. degrees in biology/biological oceanography from the University of Bremen, Bremen, Germany, in 1995 and 1999 . respectively.

From 1995 to 1999 , he was with the AlfredWegener-Institute for Polar and Marine Research, Bremerhaven, Germany. In 2002, he was with the Interuniversity Institute, Eilat, Israel, and in November 2000, he joined the Institute for Coastal Research, Helmholtz-Zentrum Geesthacht, Geesthacht, Germany. Since 2011, he has been the Head of a working group for optical remote sensing. His main research interests include optical properties of water constituents and their use for satellite and airborne remote sensing applications to study coastal waters. 